tion of $\mathrm{A} \beta$ plaques. The low $\mathrm{CH}$ diet used resulted in both significant $\mathrm{A} \beta$ plaque burden and a survivable model, allowing treatment effects after disease establishment to be assessed in the future. Extension of these technologies to an in vivo setting is practical, and should allow the study of $\mathrm{AD}$ pathogenesis in animals over time. These exciting results also hint at the promise of clinical MRI-based detection of $\mathrm{A} \beta$ plaques in humans.
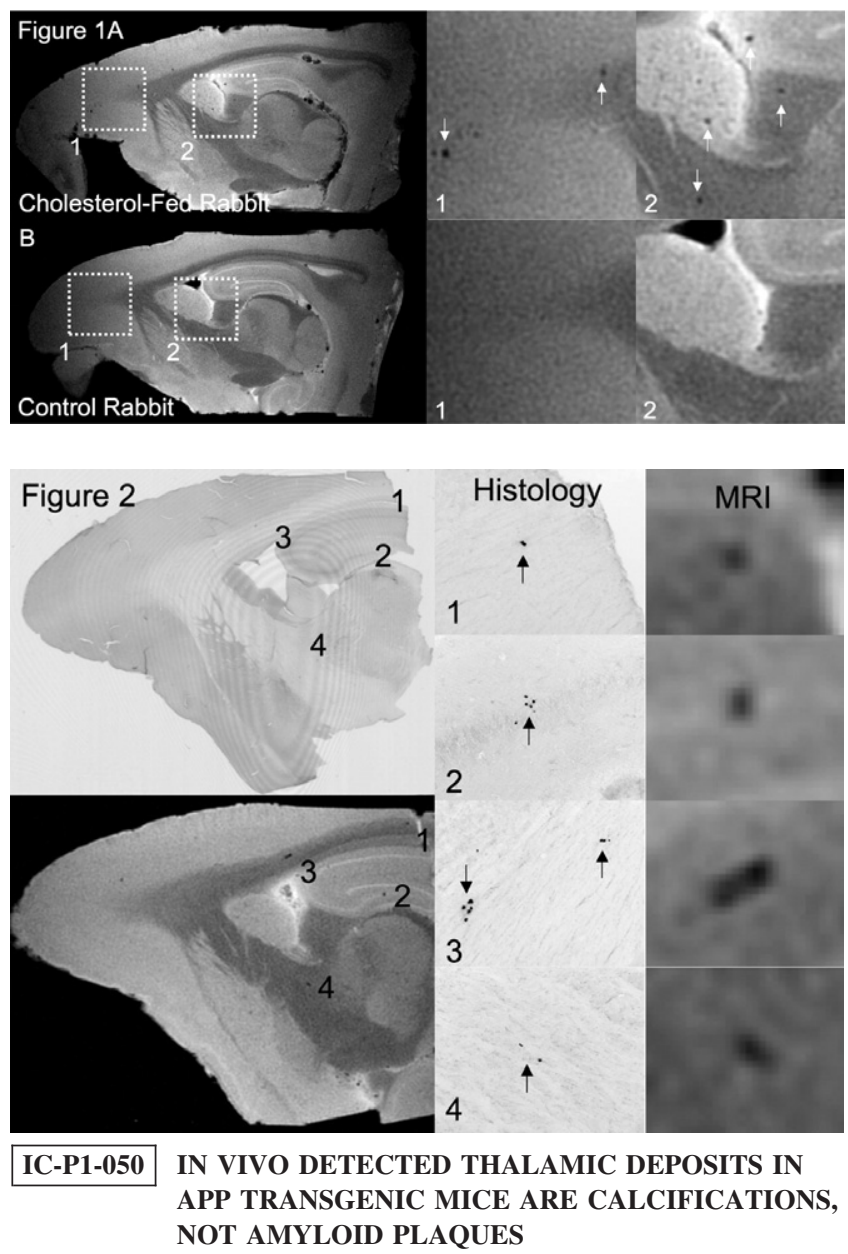

Beate Rösner ${ }^{1}$, Feng Luo ${ }^{2}$, Gerard B. Fox ${ }^{2}$, Ulrich Ebert ${ }^{1}$,

${ }^{I}$ Neuroscience Research, GPRD, Abbott, Ludwigshafen, Germany;

${ }^{2}$ Neuroscience Research, GPRD, Abbott, Abbott Park, IL, USA. Contact e-mail: beate.roesner@abbott.com

Background: Extended deposits of $\mathrm{A} \beta$ in the thalamus have been reported to be present in various strains of APP transgenic mice. We have found extended thalamic signals in 22 month old $\mathrm{Tg} 2576$ mice using $\mathrm{T} 2 *$ weighted magnetic resonance imaging $(\mathrm{T} 2 * \mathrm{MRI})$, which we then attempted to correlate with deposits identified histopathologically in the same regions. Methods: To do this we sectioned the whole brain of each animal used in MRI studies and used several staining protocols on a subset of sections to identify the nature of the deposits. Results: Located in the ventral posteromedial thalamic nucleus, the deposits were negative for amyloid deposits when stained with Congo red and negative for amyloid $\beta$ peptide when stained with the antibody $6 \mathrm{G} 1$, indicating they were not large amyloid plaques. They were also negative for $\mathrm{Fe}^{3+}$ when stained with Prussian blue, indicating they were not hemorrhages. However, all thalamic deposits stained positive for $\mathrm{Ca}^{2+}$ with Von Kossa. Interestingly, similar MRI signals were also detected in non-transgenic old mice and in another APP transgenic mouse strain at an older age. Conclusions: We conclude that the thalamic T2*-MRI signals are not large amyloid plaques but rather calcified lesions, which are observed in subcortical regions of wildtype and transgenic mice as well as AD patients and age-matched controls.

\section{IC-P1-051 AUTOMATIC DIAGNOSTIC CLASSIFICATION OF DEMENTIA WITH FDG-PET USING A SPATIAL - DECISION TREE APPROACH}

N. Sadeghi ${ }^{1}$, N. L. Foster ${ }^{2}$, A. Y. Wang ${ }^{2}$, S. Minoshima ${ }^{3}$,

A. P. Lieberman ${ }^{4}$, T. Tasdizen ${ }^{1},{ }^{1}$ School of Computing, University of Utah, Salt Lake City, UT, USA; ${ }^{2}$ Center for Alzheimer's Care, Imaging and Research, University of Utah, Salt Lake City, UT, USA; ${ }^{3}$ School of Medicine, University of Washington, Seattle, WA, USA; ${ }^{4}$ Department of Pathology, University of Michigan, Ann Arbor, MI, USA. Contact email: nedasadeghi@yahoo.com

Background: Alzheimer's disease (AD) and frontotemporal dementia (FTD) cause similar symptoms making accurate clinical diagnosis difficult. These disorders cause distinctive patterns of hypometabolism with positron emission tomography with [18F] fluorodeoxyglucose (FDG-PET). Objective(s): To develop an automated method to accurately classify FDG-PET scans in patients with AD and FTD. Methods: We used FDG-PET scans from 48 patients with pathologically confirmed $\operatorname{AD}(n=34)$ or FTD $(n=14)$ to evaluate a novel automated method for diagnostic classification. Pixels representing peak values derived from stereotactic surface projection analysis, were grouped into empirically determined regions that best separated scans from patients with AD and FTD. These regions then were used as attributes in a decision tree learning algorithm using all 48 cases. Accuracy of this approach was evaluated with leave-one-out cross validation. Results: Three decision nodes based upon hypometabolism in an arbitrarily shaped region were needed to correctly classify all 48 cases The root node classified subjects as having $\mathrm{AD}$ if there was significant hypometabolism in a region of posterior temporoparietal cortex. The other two nodes of the tree corresponded to frontal and anterior temporal areas, which are hypometabolic in FTD. Leave-one-out cross validation demonstrated the diagnostic accuracy of this method was $94 \%$. This method also had high AD sensitivity/FTD specificity (97\%), AD specificity/FTD sensitivity (86\%), positive likelihood ratio for $\mathrm{AD}(94 \%)$, and positive likelihood ratio for FTD $(92 \%)$. Conclusions: The dynamic region selection of decision tree based on the information gain is a powerful, unbiased method for classifying brain scans, requiring no prior knowledge or assumptions. This method can locate areas of abnormality for specific dementias, not easily accomplished with other classification methods, such as neural networks and multivariate analysis. This method is feasible for clinical application to increase the accurate diagnosis of AD and FTD. Acknowledgments: Supported in part by the Center for Alzheimer's Care, Imaging and Research and NIH grants AG22394, AG16976, AG08671, and AG024904.

\section{IC-P1-052 THE UTILITY OF QUANTITATIVE CORTICAL THICKNESS ANALYSIS IN INVESTIGATING BRAIN-BEHAVIOR RELATIONSHIPS IN PROGRESSIVE APHASIAS}

Daisy Sapolsky ${ }^{1}$, Akram Bakkour $^{2}$, Paige Nalipinski ${ }^{2}$, Daisy Caplan ${ }^{2}$, Bradford Dickerson ${ }^{2},{ }^{I}$ Massachusetts General Hospital, Boston, MA, USA; ${ }^{2}$ Massachusetts General Hospital, Charlestown, MA, USA. Contact e-mail:dsapolsky@partners.org

Background: Most groups of patients with progressive aphasia display heterogeneous language deficits. Even within progressive aphasia subtypes, there is frequently variability in the character and relative severity of language and behavioral abnormalities. We sought to determine whether quantitative cortical thickness analysis is useful at the individual subject level to identify anatomic correlates that relate to impairment of specific aspects of language and behavior. Methods: Individual subject quantitative analysis was performed by implementing a general linear model comparing the subject of interest to a group of controls of similar age. The location and magnitude of regional cortical thinning was obtained from MRI data and 